\title{
Improved Dose Regimen in Pediatric PET
}

\author{
Roberto Accorsi ${ }^{1,2}$, Joel S. Karp ${ }^{2}$, and Suleman Surti ${ }^{2}$ \\ ${ }^{1}$ Department of Radiology, Children's Hospital of Philadelphia, Philadelphia, Pennsylvania; and ${ }^{2}$ Department of Radiology, \\ University of Pennsylvania, Philadelphia, Pennsylvania
}

PET image quality depends strongly on patient weight and habitus, decreasing for increasing weight and body mass index. Common adult injection rules prescribe either a dose proportional to weight or a fixed dose. In light patients, image quality may improve for decreasing weight more than by inverse proportion. If better quality than in average-adult studies does not justify the associated dose burden, attractive options are to reduce scan time, reduce dose, or any combination of the 2 . The objective of this study was to determine quantitative injection rules for pediatric PET allowing clinical implementation of these trade-offs. Methods: Literature methods combining phantom with clinical data were followed to derive patient-specific noise-equivalent count rate density (NECRD) curves as a function of injected dose. From these, it was possible to estimate retrospectively for each patient the scan time that would have been sufficient for the same NECRD as in a 70-kg reference adult; the reduced dose sufficient for constant NECRD and scan time; and a general relationship among scan time, dose, and NECRD. Correlation to the patient statistic giving highest correlation, which was found to be weight, provided rules applicable prospectively. Data from 73 patients (weight, 11.5-91.4 kg; mean, $45.4 \mathrm{~kg}$ ) were acquired and analyzed. Results: Following the clinical injection rule, which was proportional to weight, the NECRD increased linearly with decreasing weight. The expression exp[0.019 $\times$ (weight $[\mathrm{kg}]-70)]$ for the time reduction possible with the current dose at constant NECRD correlated well with data $\left(R^{2}=0.86\right)$. The dose (in $\mathrm{MBq}$ ) necessary for constant NECRD that should be injected 60 min before imaging is predicted well by $14.8 \times$ $\exp [0.046 \times$ weight $(\mathrm{kg})]\left(R^{2}=0.88\right)$ with the current scan time. A more complex expression to convert NECRD in whole or part to both dose and time savings was also derived. Comparison to common pediatric injection rules showed reasonable agreement with Clark's rule, albeit not at all weights. Conclusion: Results suggest that pediatric PET of constant image quality (in an NECRD sense) can be performed with time or dose savings, up to $50 \%$ for the lightest patients $(10-20 \mathrm{~kg})$.

Key Words: PET; ALARA; dose optimization; ${ }^{18} \mathrm{~F}-\mathrm{FDG}$

J Nucl Med 2010; 51:293-300

DOI: 10.2967/jnumed.109.066332

$\mathrm{t}$ is well known that whole-body ${ }^{18} \mathrm{~F}$-FDG PET image quality depends on patient weight and habitus, decreasing with increasing weight and body mass index (BMI; the

Received May 20, 2009; revision accepted Oct. 7, 2009.

For correspondence or reprints contact: Roberto Accorsi, via Ausonio

3, 20123 Milano, Italy.

E-mail: raccorsi71@gmail.com

COPYRIGHT @ 2010 by the Society of Nuclear Medicine, Inc. ratio of weight [expressed in $\mathrm{kg}$ ] to the square of the height [expressed in $\mathrm{m}$ ]) because of both the increasing attenuation of $511-\mathrm{keV}$ photons in the patient and the increasing scatter and random fractions. The literature has understandably concentrated on the relatively common case of heavy patients, for whom poorer image quality is expected. Recent studies have suggested that in this case best imaging is not obtained with ever-increasing doses but, above a certain weight, by prolonging the duration of the scan (1).

The Children's Hospital of Philadelphia recently installed a Gemini 16 PET/CT scanner (Philips Health Care) (2). The unit is dedicated to pediatric imaging. For a 1-h uptake period, the injection protocol suggested by the manufacturer for this fully 3-dimensional gadolinium oxyorthosilicate scanner is $5.18 \mathrm{MBq} / \mathrm{kg}(0.14 \mathrm{mCi} / \mathrm{kg})$, with a minimum dose of $74 \mathrm{MBq}(2 \mathrm{mCi})$ and up to a maximum dose of $444 \mathrm{MBq}(12 \mathrm{mCi})$. This protocol is consistent with dose regimens for adults, which historically have been based more on radiation dose restrictions than on optimal use of the imaging equipment (3). The goal of this study was to investigate the implications of this injection protocol in a pediatric population.

The determination, and even the definition, of optimal performance is a particularly difficult task in medical imaging, especially when practicality is considered (3). Several metrics, from simple signal-to-noise measurements to labor-intensive human observer studies, have been used. The noise equivalent count rate (NECR) is essentially a ratio of true counts to all types of counts and accounts for the effects of Poisson statistics and those of scatter and random coincidences (4). By its definition, the NECR is a metric of data, rather than image, quality; tellingly, it operates only on sinogram data and, thus, is independent of image reconstruction. Nevertheless, the NECR has been extensively used as a proxy of image quality and has become part of the performance- and acceptance-testing procedures recommended for PET scanners (5) because it provides an established compromise between representativeness of performance and practicality of calculation.

Standard NECR measurements of whole-body scanners rely on a reference phantom meant to simulate an average, $70-\mathrm{kg}$ adult patient (5). NECR curves, however, are different for different phantoms and, thus, for different patients; this is a problem that is exacerbated in pediatric imaging, for which patients range from young adults (our heaviest 
patient was a $130-\mathrm{kg} 18$-y-old) to newborns (as young as 3 wk old, for example, in a congenital hyperinsulinism population; Jager et al. provide a recent review (6)). Unfortunately, direct measurement of the NECR curve of each patient would require both an unethically high dose and a clearly impractical scanning time (more than several hours).

Other investigators have considered the use of anthropomorphic phantoms (7) (not immediately applicable to pediatric imaging (1)) and computational approaches, some involving digital phantoms of remarkable anatomic accuracy $(8,9)$. In a recent paper, Watson et al. have described and applied to adult imaging a method to produce patientspecific NECR curves retrospectively by combining experimental phantom data with patient-specific clinical data (1).

In the present study, Watson's method was applied to a pediatric population, one for which excellent NECR is expected because of reduced attenuation and for which every opportunity for time and dose savings is particularly attractive. Interestingly, Watson et al. explicitly excluded neck and head scans from their analysis because of their consistently higher NECR and suggested that these cases could be considered for a separate study (1). Our motivation is further strengthened by the recently renewed emphasis put on the as-low-as-reasonably-achievable (ALARA) principle (10) by the Society for Pediatric Radiology ALARA conference series (11) in the specific context of pediatric radiology. Application of the ALARA principle forces the question of whether the benefit of an expected image quality superior to adult studies, which is already excellent in patients of average weight (12), is justifiable when considered against the risks of the increased dose to an especially radiosensitive population $(10,13,14)$ or of prolonged sedation or anesthesia. For this reason, we sought to derive injection regimens that effectively trade the expected NECR increase in whole or in part for a reduced dose burden, a reduced scan time, or any combination of the previous. These regimens provide the dose to be injected in terms of the weight of the patient and are the main result of this current study.

\section{MATERIALS AND METHODS}

\section{NECR and NECR Density (NECRD) Curves}

In this section a brief, nonmathematic description of the literature method used to obtain the patient-specific NECR and NECRD curves is given for the reader's convenience. Readers interested in its full details are referred to Watson et al. (1).

The technique decomposes the NECR into object-independent and object-dependent components: the former are functions of the count rate and are obtained in phantom experiments, and the latter are scaling coefficients and are calculated retrospectively from available clinical data. The combination of the 2 components results in a patient-specific whole NECR curve (i.e., the NECR as a function of injected dose). The NECRD curve is obtained by dividing the NECR by the number of pixels that define, in each frame, the volume of the patient, which is obtained from the transmission data acquired for attenuation correction.

NECR and NECRD curves can be analyzed to infer retrospectively the strategy that should have been followed to implement the dosing regimen of interest. Because this analysis shows strong correlations $\left(R^{2}>0.8\right)$ between PET physics parameters (such as NECR, dose, and acquisition time), which are known only a posteriori, and patient statistics (such as weight, height, and girth), it is possible to derive injection rules that calculate the dose based on the latter and, thus, can be used prospectively.

\section{NECRD Curve Analysis}

Figure 1A shows the shape of the NECRD curves of 2 different patients. As dose increases beyond a certain level, the total
FIGURE 1. Sample NECRD curve analysis. (A) NECRD curves for adult patient $(69.9 \mathrm{~kg})$ and for lighter patient (38 kg). (B) NECDs for 3 different scan times are shown for $69.9-\mathrm{kg}$ patient's curve. (C) Calculation of dose needed to image lighter patient with same NECRD (image quality) obtained in adult patient. (D) Calculation of time reduction possible if clinical dose is used and imaging is performed at constant NECD. $\mathrm{P}_{\text {peak }}=$ maximum NECRD; $\mathrm{P}_{90 \%}=$ point with $90 \%$ of peak NECRD; $\mathrm{P}_{\mathrm{c}}=$ dose injected according to clinical protocol (5.18 MBq/kg, 1-h uptake).
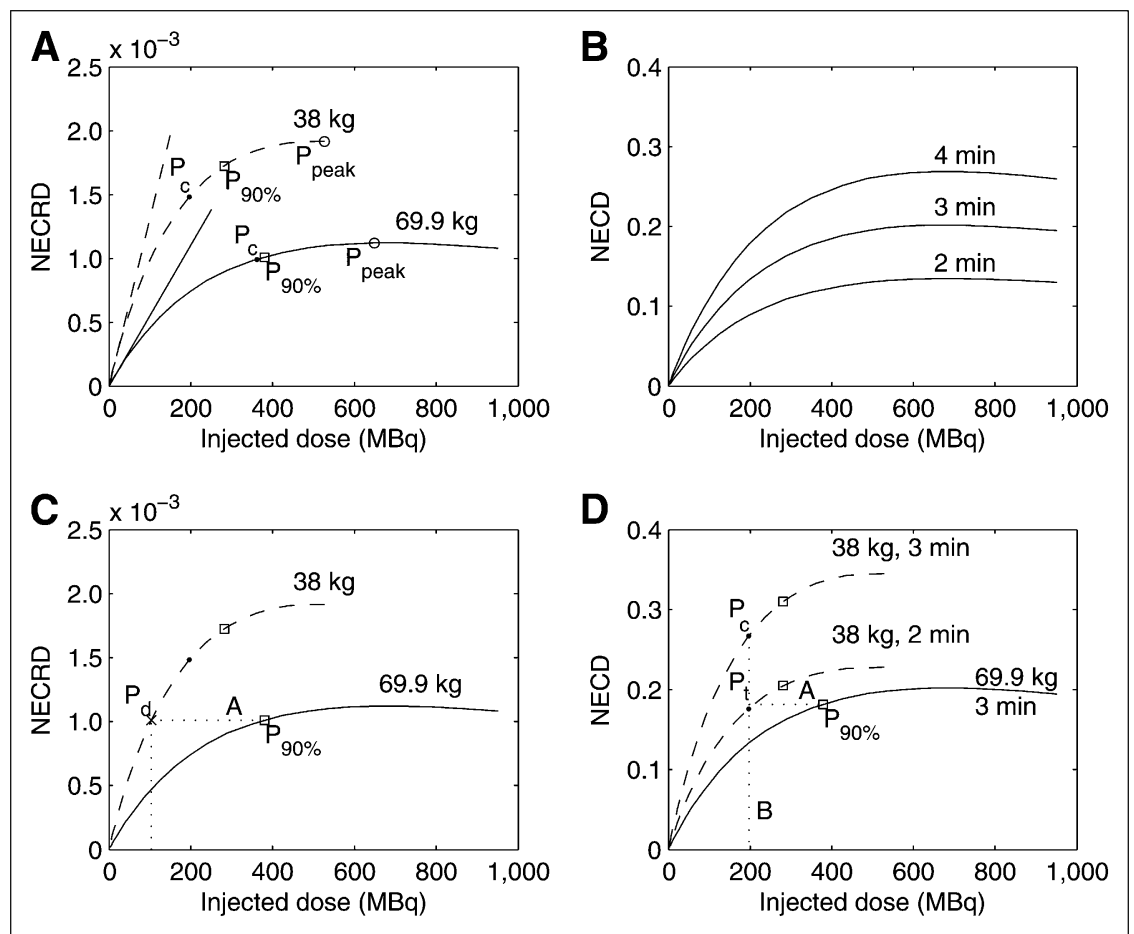
increase in NECRD is no longer proportional to the dose. More specifically, as increasing doses result in decreasing increments in NECRD, the collection of useful events becomes progressively less efficient. This is essentially due to increasing dead-time and random events. Eventually a point is reached, the maximum of the NECRD curve, after which larger injections actually cause the collection of ever fewer useful events. Another typical feature of NECRD curves is their rather flat shape around the maximum.

Image quality (in a NECR sense) is related to the NECRD curve and to the length of the scan. The quantity of interest is in fact the noise-equivalent count density curve (NECD), which is obtained from the NECRD curve by multiplying by the scanning time-if the scanning time is short, compared with the half-life of the isotope, which is the case herein. Time is simply a scaling factor and does not change the shape of the NECRD curve (Fig. 1B).

The dependence on patient weight and habitus is more complex. In lighter patients, decreased attenuation and scatter result in the collection of more useful events for the same activity. This fact is reflected in a change in the shape of the NECRD curve, which rises from zero more sharply with activity. The increased count rate, however, also results in an increased random coincidence rate and an earlier onset of dead-time effects. Figure 1A also shows the case of a 38-kg patient, whose NECRD curve starts with a steeper slope and peaks at a lower dose than an adult patient's.

A common method to determine injection rules is to choose the dose that corresponds to a large fraction $(90 \%-95 \%)$ of the peak NECRD because the sacrifice of only $5 \%-10 \%$ of image quality results in an approximate halving of the radiologic dose. However, this logic essentially optimizes scanner throughput (i.e., minimizes scan time); minimization of patient dose would require calculating the minimum dose compatible with a given scan time and an acceptable NECD level. This dose could be lower than the dose that results in $90 \%$ NECRD.

Figure 1C shows the calculation of the dose that for the same scan time would result in the same NECR as in a heavy patient. The horizontal line A starting from $\mathrm{P}_{90 \%}$ on the curve for the heavier patient intersects the NECRD curve of the lighter patient at point $P_{d}$, whose abscissa is the dose sought.

Figure 1D shows how the scan time reduction that results in constant image quality for the same dose is calculated. A vertical line $B$ is drawn from the point $P_{c}$ on the NECRD curve of the light patient. The horizontal line $\mathrm{A}$ drawn from the point $\mathrm{P}_{90 \%}$ on the NECRD curve of the adult patient intersects $B$ in $P_{t}$. The time needed for the scan of the lighter patient to match the NECD of the scan of the heavier patient is given by the time of the latter multiplied by the ratio of the ordinates of $P_{t}$ and $P_{c}$. In the particular case shown, scan time can be reduced by about one third, as demonstrated by the rescaled NECRD curve, which crosses the line A in correspondence to the clinical dose.

For each patient, it is possible to calculate a NECRD curve at each bed position (Fig. 2). Because it makes little sense to optimize injection for each position, NECRD curves were averaged over bed positions to obtain a single NECRD curve for each patient.

\section{Patient Population}

This study aimed at including all patients undergoing wholebody ${ }^{18} \mathrm{~F}$-FDG scanning, independent of clinical indication. The protocol for whole-body scanning at The Children's Hospital of Philadelphia comprises 2 parts: scanning from the mid thighs to

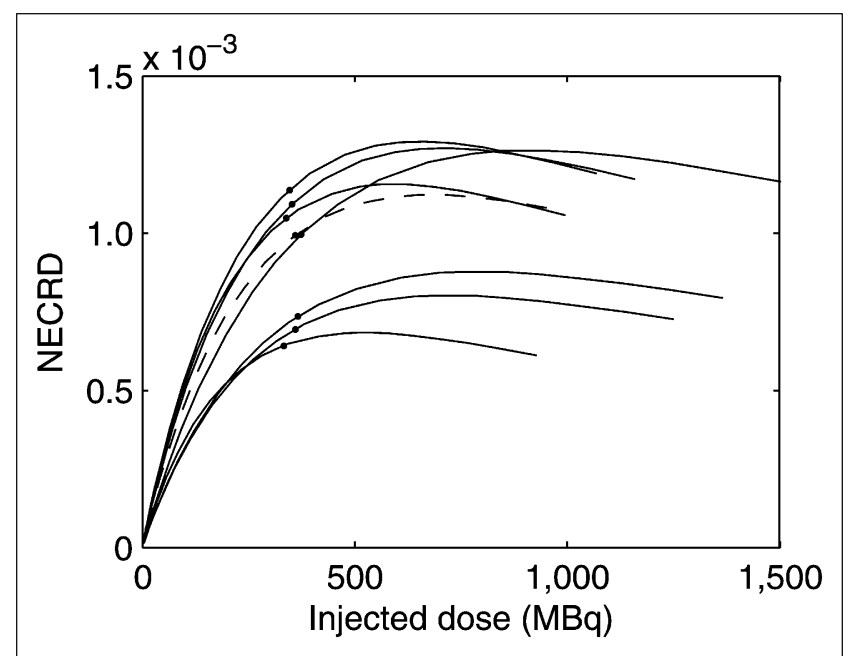

FIGURE 2. NECRD curves for each bed position of 76-kg patient. Dashed line indicates average NECRD curve. Dots indicate dose that should have been injected $1 \mathrm{~h}$ before beginning of scan of each frame to have same dose as in clinical situation.

the base of the neck, with arms above the head, followed by a scan from the base of the neck to the eyes, with arms alongside the body. Only the first part of the scan was considered. For patients who underwent longer scans, in which the legs were included, only frames from the mid thigh to the eyes were considered. Patients undergoing imaging performed in the brain acquisition mode of the scanner, which concentrates spatial sampling on a smaller field of view, were excluded. Newborns can and are imaged in brain mode even for whole-body studies.

Seventy-three patients were included. Their weight ranged from 11.5 to $91.4 \mathrm{~kg}$, with a mean of $45.4 \mathrm{~kg}$ and a median of $48.3 \mathrm{~kg}$. This population covered the range from patients who can be considered adults for PET physics purposes, who provided validation for the methods, to a much lighter population.

Consent was obtained from patients, families, or both as mandated by the Institutional Review Board of The Children's Hospital of Philadelphia on approval of the study.

\section{Derivation of Injection Rules}

The analysis of NECRD curves described above produces for each patient a minimum dose and a minimum time for constantNECD imaging. For each patient, these values can be calculated only a posteriori. The correlation of the minimum dose (and time) to patient statistics such as weight, height, girth, and BMI was verified in search of the best predictor. Injection rules were then derived by a least-mean-square error fit of a straight line to the data points or their logarithm.

A more complex procedure had to be followed for the determination of a formula capable of handling a combination of dose and time reduction and an incomplete trade-off of the excess NECD. Its derivation is detailed in the Appendix.

Several injection rules have been proposed over the years for use in pediatrics. Most rely on scaling the adult dose according to age (Young's $(15,16)$, Webster's (15), and Solomon [or Fried's] rule (16)), weight (Clark's $(15,16))$, or body surface area (BSA; Clark's area rule $(15,16))$, for which several correlations have been used 
(DuBois and DuBois (17), Mosteller (18), Boyd (19), Gehan and George (20), Haycock et al. (21), and many others). Pediatric doses, which were then compared with those derived from NECRD analysis, were derived using the rules reported in Table 1.

\section{RESULTS}

\section{NECD and Clinical Injection Regimen}

Figure 3A shows the relevant part of NECD curves and the points of clinical operation for all patients. Figure $3 \mathrm{~B}$ shows the NECD measured for each patient in the clinical scans $\left(\mathrm{P}_{\mathrm{c}}\right)$ as a function of weight; the ordinate of the points is the same as in Figure 3A. Figure 4 shows, for each patient, the NECD of these points as a fraction of the $90 \%$ peak NECD in the same patient.

It is apparent that the current injection regimen produces better image quality in lighter patients rather than constant NECD. More specifically, weight and clinical NECD correlate well $\left(R^{2}=-0.82\right)$. The following sections quantify the different ways of trading this advantage.

\section{Determination of Different Dosing Regimens: Constant NECD and Dose and Scan Time Reduction}

A reference NECD was set, selecting an arbitrary weight representative of an adult patient $(70 \mathrm{~kg})$ and calculating the NECD of the patient with the closest weight $(69.9 \mathrm{~kg})$. For the same dose regimen, it is still possible to image at reference NECD at all weights lighter than reference if the scan time is reduced. The scan time reduction $\tau$ is given by the ratio of the reference NECD and the clinical NECD of each patient. To obtain a general relationship, the line $c_{1}$ $w(k g)+c_{2}$ was fitted to the natural logarithm of this ratio. Numerically:

$$
\tau=\frac{e^{c_{1} w(k g)+c_{2}}}{e^{c_{1} 70+c_{2}}}=e^{c_{1}[w(k g)-70]}=e^{0.019[w(k g)-70]}, \text { Eq. } 1
$$

which gives $R^{2}=0.86$ and is shown in Figure 3D. For example, a $50-\mathrm{kg}$ patient can be scanned in $\exp (-0.019 \times$ $20)=68 \%$ of the time used for a $70-\mathrm{kg}$ patient while maintaining the same NECD and clinical injection protocol. If clinical scans are performed at 3 min per bed position, a 50-kg patient can be scanned for about 2 min per bed position.

This calculation implicitly assumed a complete translation of the image quality advantage to reduced scan time. Different formulas hold if the dose regimen is different or time or NECD are not constant.

\begin{tabular}{ll}
\hline \hline TABLE 1. Pediatric Dosing Rules \\
\hline \multicolumn{1}{c}{ Rule } \\
\hline Area & \multicolumn{1}{c|}{ Formula } \\
\hline Clark & $\frac{\mathrm{BSA}\left(\mathrm{m}^{2}\right)}{1.73} \times$ adult dose \\
Solomon (Fried) & $\frac{\text { Weight }(\mathrm{lbs})}{150} \times$ adult dose \\
\hline Webster & $\frac{\mathrm{Age}(\mathrm{mo})}{150} \times$ adult dose \\
\hline Young & $\frac{\mathrm{Age}(\mathrm{y})+1}{\mathrm{Age}(\mathrm{y})+7} \times$ adult dose \\
\hline
\end{tabular}

Determination of Different Dosing Regimens: Constant NECD and Scan Time and Dose Reduction

The calculation of a full translation to dose reduction is illustrated in Figure 3C, which also plots weight as a function of the dose that results in imaging at reference NECD, as calculated in Figure 3A. The logarithm of the dose data points as a function of weight can be fitted with a straight line $\left(R^{2}=0.88\right)$, giving the relationship

$$
\operatorname{dose}(\mathrm{MBq})=14.8 \times \exp [0.046 \times \text { weight }(\mathrm{kg})] . \quad \text { Eq. } 2
$$

A straight line was found to correlate well with the doses injected clinically $\left(\right.$ dose $[\mathrm{MBq}]=4.60 \times w+3.37 ; R^{2}=$ 0.99 ) and is shown in Figure $3 \mathrm{C}$, which also shows the dose for $90 \%$ NECR.

A first-degree polynomial was also fitted to the ratio of the dose necessary for constant NECRD and the dose used clinically, calculated for each patient, to obtain an estimate of the dose reduction $\left(R^{2}=0.68\right.$, which improves only slightly to 0.69 with higher-degree polynomials and to 0.72 after logarithmic transformation). The value of the polynomial was normalized to 1 at the reference weight to eliminate the approximate $10 \%$ inconsistency between the fitting polynomial and the reference data point due to data scatter, yielding $d / d_{r}=0.0113 w+0.21$. This curve is also shown in Figure 3D.

\section{Determination of Different Dosing Regimens: Constant Quality, Scan Time, and Dose Reduction}

NECD can be traded for both a scan time and a dose reduction, neither of which is as large as indicated in the previous 2 sections.

To obtain the time reduction for a given dose, Equation 1B can be solved for $\tau$ :

$$
\begin{aligned}
\tau= & \operatorname{NECRD}\left(d_{r}, w_{r}\right) /\left[a(w) d^{2}+b(w) d+c(w)\right] \\
= & {\left[-5.9 \cdot 10^{-5} \exp (-0.039 \times w) d^{2}+5.1 \cdot 10^{-2}\right.} \\
& \exp (-0.036 \times w) d+0.33 \exp (-0.030 \times w)]^{-1},
\end{aligned}
$$

Eq. 3

where $d$ is the desired dose, in megabecquerels, which may be given by the clinical regimen or be lower. In the former case, because of data scatter, Equation 3 is logically equivalent to Equation 1, but not numerically.

If instead the desired time fraction $\tau$ is known, Equation $1 \mathrm{~B}$ must be solved for $d$. The dose sought is given by the minimum of the 2 roots of the second-degree polynomial:

$$
\begin{aligned}
& -5.9 \cdot 10^{-5} \exp (-0.039 \times w) d^{2}+5.1 \cdot 10^{-2} \\
& \exp (-0.036 \times w) d+0.33 \exp (-0.030 \times w)-1 / \tau=0 .
\end{aligned}
$$

Eq. 4

If $\tau$ is set to 1 (i.e., no time reduction), the same result as in the previous section, in which all advantage was 

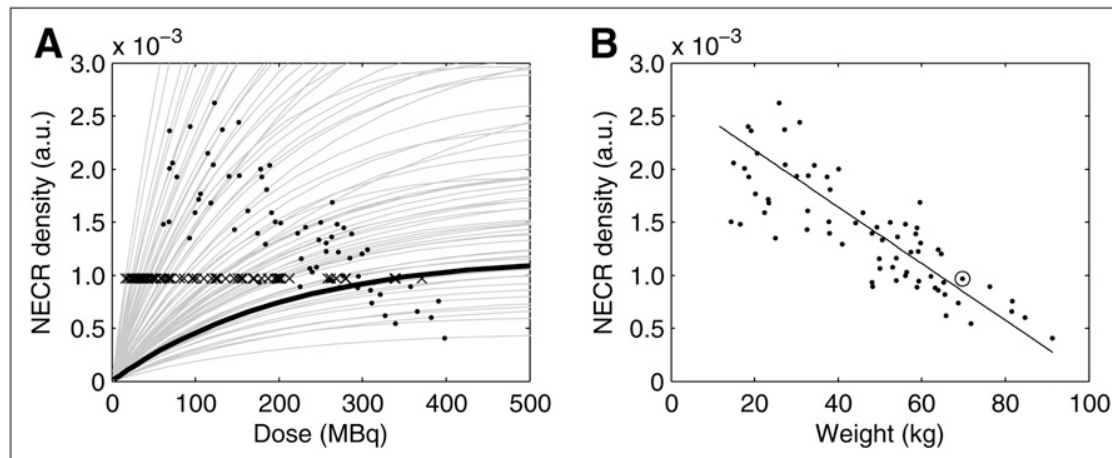

FIGURE 3. (A) NECRD curves for all patients. Thick line indicates patient assumed to represent average adult patient $(69.9 \mathrm{~kg})$. Dots indicate clinical dose. Crosses indicate dose that in each patient would have produced same NECRD as in reference patient. (B) Each point indicates weight and
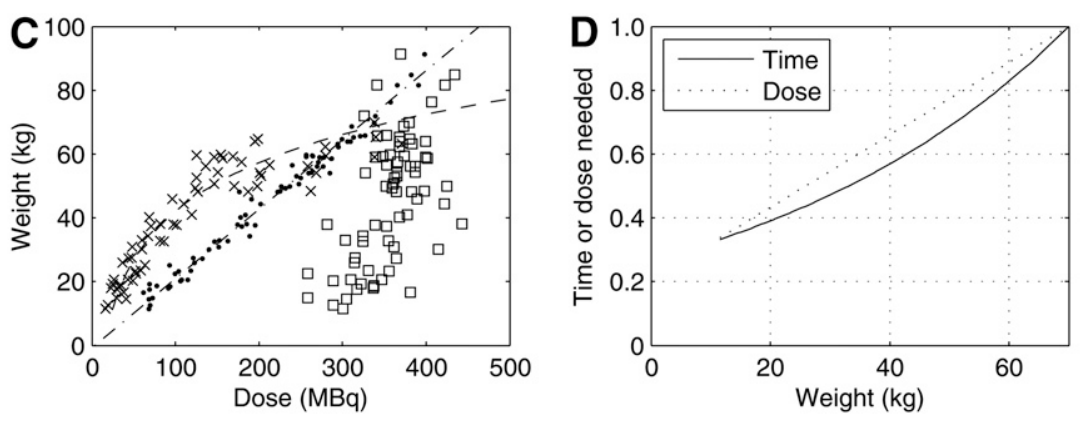
NECRD of patient in clinical scan. Circled point represents average adult patient. Dots indicate clinical dose. (C) Each cross represents weight and dose derived in A for each patient. Dashed line represents Equation 2. Dash-dot line indicates clinical injection protocol. Squares indicate dose for operation at $90 \%$ peak NECRD. (D) Time (solid) and dose (dotted) reductions relative to scan parameters for reference adult patient. a.u. = arbitrary unit.

converted to dose, is logically obtained but not numerically, again because of data scatter.

It is also possible to trade only part of the excess NECD; this is conveniently done using the desired value of $\operatorname{NECRD}\left(d_{r}, w_{r}\right)$. For example, to image with NECRD $20 \%$ larger than in the reference case, it is sufficient to use $\operatorname{NECRD}\left(d_{r}, w_{r}\right)=1.2 \times 8.3 \cdot 10^{-4}=9.96 \cdot 10^{-4}$. Equations 3 and 4 are modified accordingly.

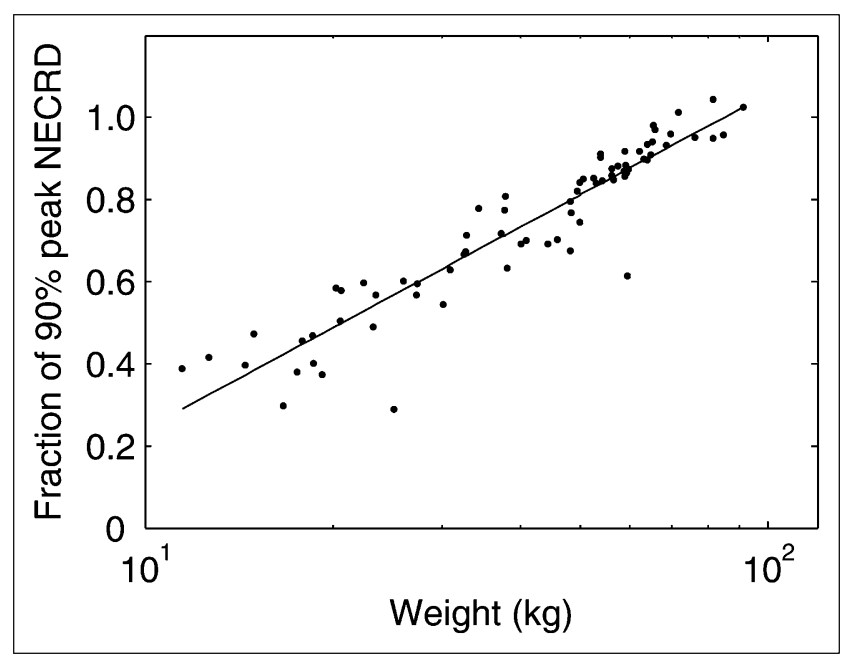

FIGURE 4. Fraction of $90 \%$ of peak NECRD as function of weight after clinical injection protocol, that is, $P_{c} / P_{90 \%}$. Data show good correlation with logarithm of patient weight $\left(R^{2}=\right.$ 0.94). Least-mean-square fit line is $P_{c} / P_{90 \%}=0.355$ $\log (w(\mathrm{~kg}))-0.576$. Scanner is working at or about $90 \%$ peak NECRD for patients having reference weight $(70 \mathrm{~kg})$.

\section{Correlation to Patient Statistics Other Than Weight}

Weights retrieved from PET file headers and girths, which were measured as the total number of pixels subtending each patient in the transmission scan, were available for all patients. Because all bed positions cover the same axial extent, this is more properly a measure of patient cross-section, averaged over the axial field of view covered at each bed position. Heights were retrieved from patient charts but were not always available. For the 59 of 73 patients whose height was known, correlation of the dose for constant NECD and imaging time to weight, height, girth, and BMI was attempted. The correlation coefficient was $R^{2}=0.86,0.69,0.81$, and 0.82 , respectively. For this reason, weight was identified as the best single predictor of optimal dose when imaging at constant NECRD and time.

\section{Comparison to Other Pediatric Dosing Rules}

Several rules have been proposed over time to calculate pediatric from adult doses. The 5 popular rules given in Table 1 were applied to derive doses for patients whose data were available. For each patient, the weight was plotted in Figure 5 against these doses to provide a comparison to the results of NECRD analysis.

\section{DISCUSSION}

Figure 1A verifies that the clinical injection protocol results in operation at or close to $90 \%$ peak NECRD for an adult-sized patient. This is generally true for patients weighing $60 \mathrm{~kg}$ or more (Fig. 4). In lighter patients, imaging occurs at a much lower fraction of the peak NECRD. Thus, for these patients it is possible to increase the injected dose without saturating the scanner. This 
FIGURE 5. Comparison of adult dose (dash-dot) and dose derived from NECRD analysis (dashed) with dose derived for individual patients from injection rules given in Table 1. (A) Area rule $(B S A)$ based on DuBois and DuBois formula. (B) Clark's formula. (C) Solomon's formula. (D) Young's $(\triangle)$ and Webster's $(\diamond$, also known as Young's modified rule) formula.
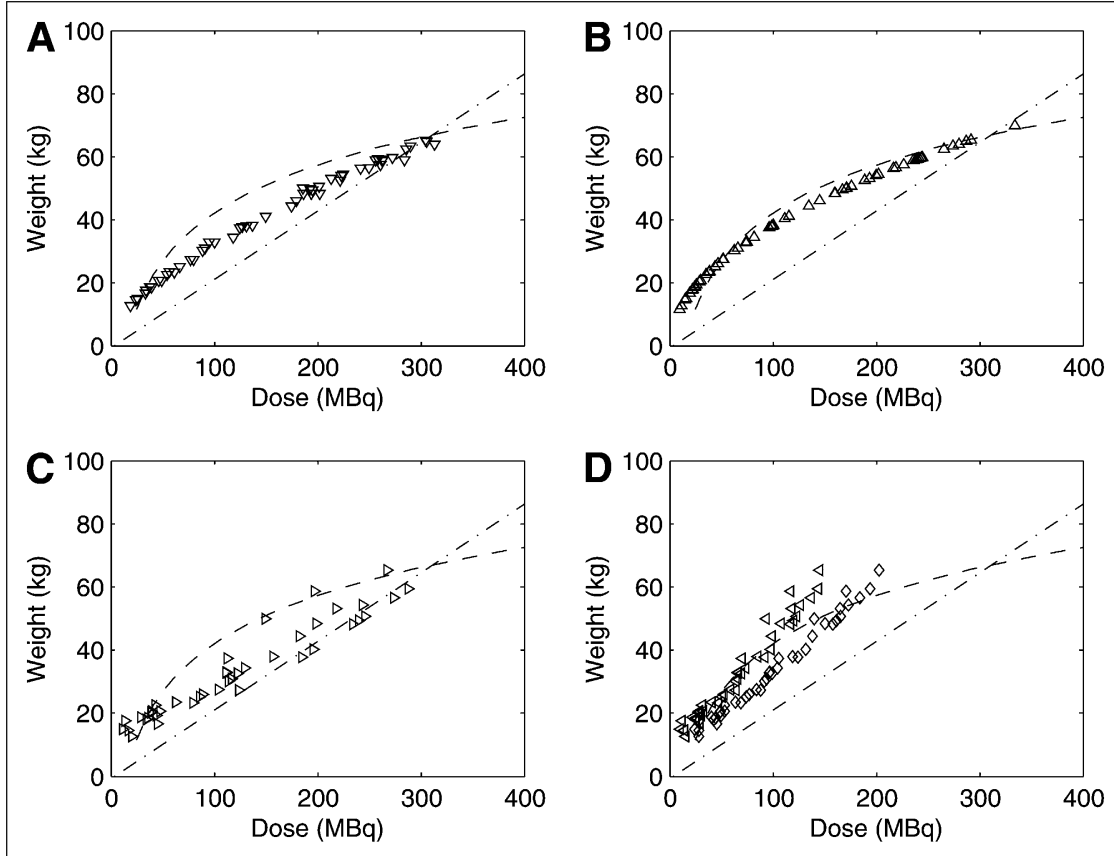

choice would minimize scan time and improve patient flow but also increase the radiologic dose. However, the current clinical dose already achieves in lighter patients a much higher absolute value of the NECRD than in adults (Fig. $3 \mathrm{~A})$. Thus, when scanning a light patient for as long as the reference patient is scanned, which is the current clinical practice, a higher NECD is expected. If constant image quality is sought, the dose can be reduced. Figure $1 \mathrm{C}$ shows the specific case of one such patient. Figure 3 extends this analysis to all patients. For patients heavier than the reference scan, line A (Fig. 1C) does not usually intersect the NECRD curve. This is consistent with the observation that, for such patients, constant image quality can be achieved only by scanning for a longer time (1). These patients were necessarily not included in the calculation of time and dose reductions.

Figure 3D summarizes the results: imaging at constant NECD can be achieved with a dose or time reduction roughly proportional to weight, which amounts to about $50 \%$ for a patient weight of $20 \mathrm{~kg}$.

As it should be expected, clinical doses were found to correlate highly with weight. However, the slope (4.60 $\mathrm{MBq} / \mathrm{kg}[0.124 \mathrm{mCi} / \mathrm{kg}]$; Fig. $3 \mathrm{C})$ is different from the recommended $5.18 \mathrm{MBq} / \mathrm{kg}(0.14 \mathrm{mCi} / \mathrm{kg})$. This is consistent with an excess wait of about $20 \mathrm{~min}$. The average PET scan involves 5.2 frames, so that the average frame is imaged almost $8 \mathrm{~min}$ after the start of the PET scan. The rest of the delay can be attributed to the acquisition of topograms, scan planning, and low-dose CT acquisition, which precede PET.

When the clinical dose is substituted in Equation 3, the time saving $\tau$ obtained should be, in principle, the same as obtained from Equation 1. In practice, the results are not exactly the same, again because of scatter in the data. The 2 expressions correlate well $\left(R^{2}=0.95\right)$ and have a meansquare relative error of about $10 \%$ over the entire weight range. Similarly, when $\tau=1$ is used in Equation 4, the result is not exactly the same as for Equation 2, but the mean-square relative error is about $10 \%$. These results appear to cross-validate the methods and provide an estimate of the accuracy that can be expected from the formulas developed.

The approach of fitting a first-degree polynomial to the ratios of the dose necessary for constant NECRD and the clinical dose results in a smoother curve than does taking the ratio of the polynomials fitting these doses, that is, Equation 2 and the clinical dose algorithm. Data scatter explains the difference of the 2 curves (not shown).

Overall, the choice of first-order polynomials seemed appropriate for the simplicity of the results, whose lack of fine details is less suggestive of features that cannot be supported given the scatter in the data points. As a rule of thumb, because the correlation coefficient between weight and NECD is $R^{2}=0.86$, accuracy better than about $15 \%$ should not be expected from any relationship. The variability over bed positions to be expected in individual patients (exemplified in Fig. 2) further cautions against attributing too much accuracy to the results presented.

As duly indicated in the "Materials and Methods" section, average NECRD curves were considered. The analysis was repeated considering single curves for validation. Results are entirely analogous, but for a more effective presentation average data were used.

Equation 4 has been verified to fit reasonably the population data for fractions of the reference time and dose as low as $40 \%$ and for weights between 10 and $80 \mathrm{~kg}$. Outside this range, the formula will yield extrapolated results that should be taken with further caution. 
Figure 5 shows that NECRD analysis corrects adult doses in the same direction as that suggested by the pharmacologic rules of Table 1, which were derived from different considerations. The extent of the corrections can be similar over certain weight intervals but in general is different. Remarkably, Clark's rule, being based on weight, results in a smooth curve; further, good overall agreement is found, but differences by as much as $50 \%$ can be found for the lightest patients. Solomon's rule seems to agree more with the adult dose, and the area rule (in its version based on the DuBois and DuBois formula for BSA) is somewhere in between. The NECRD prediction agrees well with Young's rule up to about $40 \mathrm{~kg}$ and generally yields a lower dose than Webster's rule.

Pediatric doses are used commonly but rely on an assumption that children are small adults, whereas it is well known that their metabolism is qualitatively different (22). The doses derived herein do not rely on such an assumption.

The NECRD summarizes in 1 figure of merit the most basic elements of a PET acquisition. However, the NECRD depends only on how many counts are collected and the relative importance of scatter and random events: it does not account for other effects, such as those of image reconstruction or of the corrections for scatter and random events. In this sense, it would be more appropriate to state that the NECRD is a gauge of data quality, not image quality. Throughout the text, we have chosen to use the expression image quality in its common-language meaning, not in a technical sense. Despite its limitations, the NECRD has been shown to correlate at least to some extent with image quality and is widely used for the evaluation of scanner performance in view of its availability and objectivity $(2,23-25)$; by contrast, the ultimate image quality metric, human observer performance, is difficult and laborious to measure, when at all possible (3).

All results follow from a proven theoretic model based on experimental data. Nevertheless, it is advisable to implement any changes in a gradual way. In this light, PET scanners with list-mode acquisition capability provide an efficient means of verifying the predictions for time reduction with minimal or no impact on clinical care.

The injection rules derived in this article apply only to our scanner and those based on the same PET unit (e.g., Allegro; Philips Health Care). The methodology used, however, is entirely general and can be applied to other scanners to derive similar dose regimens.

\section{CONCLUSION}

When following an injection protocol proportional to weight, the NECD (data quality) of PET images was found to improve for decreasing weight from the reference case of an adult-sized (70-kg) patient. This advantage could be traded in whole or part for reduced scan time or injected dose. Equations 1 and 2 indicate the reduced time and dose that can be used when the advantage is completely transferred to time and dose, respectively, that is, imaging preserves the same NECD. Equation 4 is more complex but allows arbitrary combinations of time and dose reductions, whereas image quality is traded in whole or part.

As compared with height, girth, and BMI, weight was found to be the patient statistic correlating best with the dose necessary for imaging at constant NECD and time.

\section{APPENDIX}

The determination of a formula capable of handling a combination of dose and time reduction and an incomplete trade-off of the excess NECD starts from the observation that the NECD depends on the shape of the curve, as captured by some patient statistic (typically but not necessarily the weight $w$ ); the point of operation on the curve, ultimately governed by the injected dose $d$; and the acquisition time $t$, which works as an overall scaling coefficient. The NECD is given by the product $t \operatorname{NECRD}(d$, $w)$. The curves of Figure 1A are 2 examples of the shape of the function $\operatorname{NECRD}(d, w)$ for 2 weights. For the reference weight $w_{r}$, reference dose $d_{r}$, and reference scan time $t_{r}$, the NECD is $t_{r} \operatorname{NECRD}\left(d_{r}, w_{r}\right)$, which is the scalar measured on the clinical data of the reference patient. All trade-offs can be calculated setting $t \operatorname{NECRD}(d, w)=t_{r} \operatorname{NECRD}\left(d_{r}, w_{r}\right)$.

For example, the expression regulating the fraction of $t_{r}$ that will be sufficient to obtain the same NECD is given by $\tau=t / t_{r}=\operatorname{NECRD}\left(d_{r}, w_{r}\right) / \operatorname{NECRD}(d, w) . \operatorname{NECRD}\left(d_{r}, w_{r}\right)$ is immediately available from the data of the reference patient; however, $\tau$ still cannot be calculated because an analytic expression for $\operatorname{NECRD}(d, w)$ is not available. When a full trade-off in dose or time was possible, this problem was solved by calculating the function of $d$ alone $\operatorname{NECRD}\left(d, w_{p a t}\right)$ (i.e., the NECRD curve available for each patient [of weight $w_{p a t}$ ]), evaluating it for the dose that the clinical algorithm prescribed for weight $w_{p a t}$, and then by obtaining a correlation between $\tau$ and $w$. An alternative approach is to approximate $\operatorname{NECRD}(d, w)$ by fitting to the available experimental NECRD curves. For a given weight, the only part of the curve relevant to practical cases (i.e., that corresponding to a dose between 0 and the $90 \%$ dose) is found empirically to be reasonably fitted with a seconddegree polynomial and, thus, can be described by 3 coefficients, $a, b$, and $c$. These three coefficients can be calculated for every patient curve available, and their dependence on weight can again be summarized with 3 different exponential fits that give $a(w), b(w)$, and $c(w)$. The result of this procedure is the approximation

$$
\operatorname{NECRD}(d, w) \approx a(w) d^{2}+b(w) d+c(w), \quad \text { Eq. } 1 \mathrm{~A}
$$

in which the patient weight $w_{p a t}$ and the dose given by the injection algorithm for $w_{\text {pat }}$ can be substituted for $w$ and $d$, respectively, to obtain $\operatorname{NECRD}\left(d, w_{\text {pat }}\right)$, and hence $\tau$, for any desired patient weight. 
The form $\tau=\operatorname{NECRD}\left(d_{r}, w_{r}\right) / \operatorname{NECRD}(d, w)$, along with Equation 1A, also allows the calculation of the dose reduction for a given time reduction $\tau$, which can be calculated by taking the minimum solution of the seconddegree equation in the variable $d$ :

$$
a(w) d^{2}+b(w) d+c(w)-\operatorname{NECRD}\left(d_{r}, w_{r}\right) / \tau=0 .
$$

The other solution is not of interest because it is greater than the peak NECRD (i.e., it belongs to a dose range where the NECRD cannot be approximated with the second-degree polynomial anyway).

The coefficients $a, b$, and $c$ of Equation 1A show excellent correlation with weight $\left(R^{2}<-0.95\right)$. Their expressions are $a(w)=-4.9 \cdot 10^{-8} e^{-0.039 w(\mathrm{~kg})}, b(w)=$ $4.2 \cdot 10^{-5} e^{-0.036 w(\mathrm{~kg})}$ and $c(w)=2.7 \cdot 10^{-4} e^{-0.030 w(\mathrm{~kg})}$. These coefficients can be used in Equation 1A with $w_{r}$ and $d_{r}$ (expressed in $\mathrm{MBq}$ ) to obtain the reference $\operatorname{NECRD}\left(d_{r}, w_{r}\right)=8.3 \cdot 10^{-4}$.

\section{ACKNOWLEDGMENTS}

We are indebted to the patients who consented-often at a demanding time - and to all PET technologists at CHOP. This work was funded by the Society for Pediatric Radiology Research and Education Foundation through a Research Fellow Award (July 2007-June 2009).

\section{REFERENCES}

1. Watson CC, Casey ME, Bendriem B, et al. Optimizing injected dose in clinical PET by accurately modeling the counting-rate response functions specific to individual patient scans. J Nucl Med. 2005;46:1825-1834.

2. Surti S, Karp JS. Imaging characteristics of a 3-dimensional GSO whole-body PET camera. J Nucl Med. 2004;45:1040-1049.

3. Badawi RD, Dahlbom M. NEC: some coincidences are more equivalent than others. J Nucl Med. 2005;46:1767-1768.

4. Strother SC, Casey ME, Hoffman EJ. Measuring PET scanner sensitivity: relating countrates to image signal-to-noise ratios using noise equivalent counts. IEEE Trans Nucl Sci. 1990;37:783-788.

5. National Electrical Manufacturers Association (NEMA). Performance Measurements of Positron Emission Tomographs. NEMA Standards Publication NU 2-2001. Rosslyn, VA: NEMA; 2001.
6. Jager PL, Chirakal R, Marriott CJ, et al. 6-L- ${ }^{18}$ F-fluorodihydroxyphenylalanine PET in neuroendocrine tumors: basic aspects and emerging clinical applications. J Nucl Med. 2008;49:573-586.

7. Lartizien C, Comtat C, Kinahan PE, et al. Optimization of injected dose based on noise equivalent count rates for 2- and 3-dimensional whole-body PET. $\mathrm{J} \mathrm{Nucl}$ Med. 2002;43:1268-1278.

8. Segars PW, Tsui BMW. Study of the efficacy of respiratory gating in myocardial SPECT using the new 4-D NCAT. IEEE Trans Nucl Sci. 2002;49:675-679.

9. Dewaraja YK, Wilderman SJ, Ljungberg M, Koral KF, Zasadny K, Kaminiski MS. Accurate dosimetry in ${ }^{131} \mathrm{I}$ radionuclide therapy using patient-specific, 3-dimensional methods for SPECT reconstruction and absorbed dose calculation. J Nucl Med. 2005;46:840-849.

10. International Commission on Radiological Protection (ICRP). The 2007 Recommendations of the International Commission on Radiological Protection. ICRP publication 103. Maryland Heights, MO: Elsevier; 2008.

11. Strauss KJ, Kaste SC. The ALARA (as low as reasonably achievable) concept in pediatric interventional and fluoroscopic imaging: striving to keep radiation doses as low as possible during fluoroscopy of pediatric patients-a white paper executive summary. Pediatr Radiol. 2006;36(suppl 14):110-112.

12. Everaert H, Vanhove C, Lahoutte T, et al. Optimal dose of ${ }^{18} \mathrm{~F}-\mathrm{FDG}$ required for whole-body PET using an LSO PET camera. Eur J Nucl Med Mol Imaging. 2003;30:1615-1619.

13. Hall EJ, Brenner DJ. Cancer risks from diagnostic radiology. Br J Radiol. 2008;81:362-378.

14. Goske MJ, Applegate KE, Boylan J, et al. The Image Gently campaign: working together to change practice. AJR. 2008;190:273-274.

15. Evans K. Paediatric radiopharmacy. In: Sampson CB, ed. Textbook of Radiopharmacy Theory and Practice. Amsterdam, The Netherlands: Gordon \& Breach; 1994:328-330.

16. Churchill JA. Posology in infants and children. Dev Med Child Neurol. 1964;6:610-613.

17. DuBois D, DuBois EF. A formula to estimate the approximate surface area if height and weight be known. Arch Intern Med. 1916;17:863-871.

18. Mosteller RD. Simplified calculation of body surface area. $N$ Engl J Med. 1987;317:1098.

19. Boyd E. The Growth of the Surface Area of the Human Body. Minneapolis, MN: University of Minnesota Press; 1935.

20. Gehan EA, George SL. Estimation of human body surface area from height and weight. Cancer Chemother Rep. 1970;54:225-235.

21. Haycock GB, Schwartz GJ, Wisotsky DH. Geometric method for measuring body surface area: a height weight formula validated in infants, children and adults. J Pediatr. 1978;93:62-66.

22. White L. Foundations of Nursing. Clifton Park, NY: Thomson/Delmar Learning; 2004.

23. Mawlawi O, Podoloff DA, Kohlmyer S, et al. Performance characteristics of a newly developed PET/CT scanner using NEMA standards in 2D and 3D modes. J Nucl Med. 2004;45:1734-1742.

24. Brambilla M, Secco C, Dominietto $M$, et al. Performance characteristics obtained for a new 3-dimensional lutetium oxyorthosilicate-based whole-body PET/CT scanner with the National Electrical Manufacturers Association NU 22001 standard. J Nucl Med. 2005;46:2083-2091.

25. Erdi YE, Nehmeh SA, Mulnix T, et al. PET performance measurements for an LSO-based combined PET/CT scanner using the National Electrical Manufacturers Association NU 2-2001 standard. J Nucl Med. 2004;45:813-821. 